ournal of

\title{
You Can't Always Rely on the Imaging in Posterior Circulation Infarcts
}

Lisa Gillinder, Luke Scarborough and Simon Broadley

Gold Coast University Hospital, Southport, Queensland, Australia

\section{Correspondence to:}

Lisa Gillinder

Gold Coast University Hospital, Southport

Queensland, Australia

E-mail:1_gillinder@hotmail.com

Received: January 11, 2018

Accepted: February 20, 2018

Published: February 22, 2018

Citation: Gillinder L, Scarborough L, Broadley S. 2018. You Can't Always Rely on the Imaging in Posterior Circulation Infarcts. J Neuroimaging Psychiatry Neurol 3(1): 9-11.

Copyright: (C) 2018 Gillinder et al. This is an Open Access article distributed under the terms of the Creative Commons Attribution 4.0 International License (CC-BY) (http://creativecommons. org/licenses/by/4.0/) which permits commercial use, including reproduction, adaptation, and distribution of the article provided the original author and source are credited.

Published by United Scientific Group

\begin{abstract}
Diffusion weighted imaging is the most sensitive technique for diagnosis of acute ischaemic stroke and is often relied upon for clinical decision making. We report a case of a 20 year of female presenting with Claude's syndrome which was DWI negative on MRI performed at 6 hours post symptom onset. Repeat MRI was performed, revealing acute infarction in the Left paramedian midbrain. Secondary causes were excluded. This case highlights the importance of history and examination skills as the primary diagnostic tools for acute ischaemic stroke, with neuroimaging being utilised as an adjunct to confirm diagnosis. This is especially true in hyperacute cases with posterior circulation symptoms.
\end{abstract}

\section{Keywords}

Stroke, CVA, Posterior circulation, Cerebral infarct, Claude's syndrome, MRI, Diffusion weighted imaging, Neuroimaging

\section{Abbreviations}

AF: Atrial Fibrillation; DWI: Diffusion Weighted Imaging; ECG: Electrocardiogram; MR: Magnetic Resonance; MRI: Magnetic Resonance Imaging

\section{Introduction}

In the age of advanced neuroimaging it is easy to forget that these investigations are not $100 \%$ sensitive. Diffusion weighted imaging (DWI) is the most sensitive imaging technique for diagnosing acute ischaemic stroke and is frequently utilised to guide urgent treatment [1]. We report a case of DWI negative Claude syndrome which subsequently became positive on repeat imaging.

\section{Case Report}

A 20 year old female of Chinese ancestry presented to the emergency department with sudden onset of diplopia and poor balance causing inability to walk and falling to the right. There was no associated headache or neck pain and she was systemically well with no recent history of trauma. There was no limb weakness and no sensory changes and no background of other neurologic symptoms or other medical diagnoses. A history of alcohol excess was notable, with intake of at least 32 standard drinks per week. Untreated anxiety and depression were also significant.

Examination revealed a left pupil sparing, partial oculomotor nerve palsy 
with preservation of ocular movements in the right eye and no associated trochlear nerve palsy. The remainder of the cranial nerve examination was normal. Limb power and sensation was normal with normal tone and reflexes. There was mild incoordination in the right limbs and pronounced gait ataxia with veering to the right. Initially the patient was able to stand without assistance with eyes open. There were no haemodynamic abnormalities and ECG revealed sinus rhythm.

Magnetic resonance (MR) imaging brain was chosen over computed tomography (CT) in view of the presumed midbrain lesion and to prevent unnecessary radiation exposure in a young female. DWI and T2 Flair sequences were acquired using a 3T scanner at 6 hours post onset and revealed no abnormality (Figure 1A). Symptoms persisted but were improving. Repeat MR imaging on day 2 of admission (45 hours after symptom onset) showed an acute infarct in the left paramedian midbrain (Figure 1B). MR angiogram showed normal cervical and intracranial vessels with no evidence of vertebrobasilar dissection. Prolonged ECG monitoring showed no arrhythmia and a transoesophageal echocardiogram with bubble study showed no evidence of a patent foramen ovale or other cardiac cause of embolus. Haematological, biochemical and immunological investigations for potential causes of a pro-thrombotic state or vasculitis were negative or normal. The patient was treated with an antiplatelet agent and a statin. She was counselled regarding the need to reduce her alcohol consumption and referred to mental health services for ongoing management of her anxiety and depression.
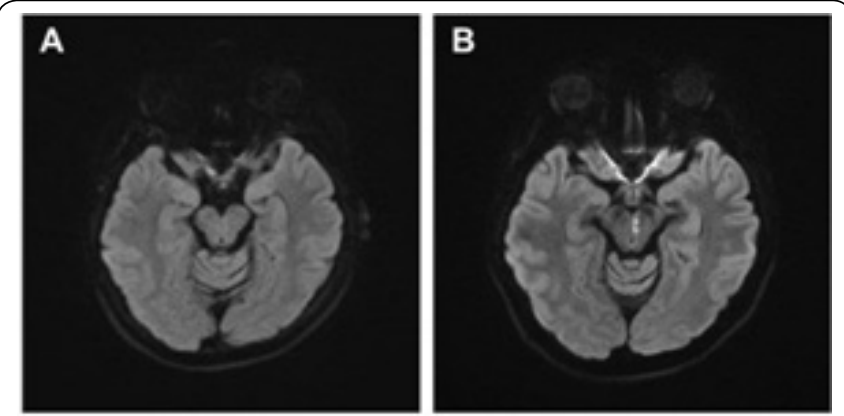

Figure 1: A) DWI at the level of the midbrain in the hyperacute phase of stroke shows no diffusion restriction. B) Repeat DWI at 45 hours post symptom onset, demonstrating diffusion restriction in the left paramedian midbrain.

\section{Discussion}

This case highlights the importance of a thorough clinical examination and familiarity with clinical stroke syndromes. The sudden onset of symptoms and the clinical picture of ipsilateral pupil sparing third nerve palsy and contralateral ataxia is classical of Claude's syndrome, caused by ischaemia in the ventromedial midbrain [2]. However, given the result of the initial MRI and the very atypical age of the patient it would have been very easy to have discounted the possibility of this event having been a stroke.

Although DWI is a very sensitive modality for detection of acute ischaemia, false negatives are not unheard of, particularly in cases of small posterior circulation infarcts
$[3,4]$. When acquired in isolation, DWI may fail to detect up to $20 \%$ of acute infarcts [5]. Even if a complete stroke protocol MRI is acquired, there may be a false negative result in up to $9.5 \%$ [6]. Lovblad et al., published one of the early reports on the diagnostic capability of this sequence and found sensitivity was $88 \%$ and specificity was $94 \%$ [7]. Furthermore, they reported that false negative rates were higher if the scan was performed within the first 6 hours after symptom onset. Since that time, many advances have been made and more recent reports regarding imaging in hyperacute infarction have shown improved diagnostic accuracy when using current $1.5 \mathrm{~T}$ scanners, but lower sensitivity and specificity when images are acquired using $3 \mathrm{~T}$ scanners [8].

False negative scans are likely attributable to either haemodynamic variability between patients and/or technical factors involved in imaging acquisition. In the ischaemic state there is accumulation of intracellular fluid and a relative reduction in diffusibility resulting in the hyperintense signal seen on DWI. It seems likely that the point at which ischaemic areas become impaired to the degree that they produce increased DWI signal could vary depending on individual haemodynamic and homeostatic factors. This is supported by studies which have found there is a differential susceptibility of brain regions to ischaemia, with the brainstem being relatively resistant. The reasons for this are unclear but are possibly related to differences in spreading depolarisations and $\mathrm{Na}^{+} / \mathrm{K}^{+}$pump function in the brainstem [9]. Furthermore due to the mechanism of posterior circulation infarcts, the clinical symptoms can fluctuate, and this difference might also explain delayed MRI findings [10].

Technical factors such as poor spatial resolution, high signal to noise ratios and susceptibility artefact which is especially troublesome in the posterior fossa can complicate matters further. This would not explain a negative result in the case presented as the technical quality of the initial scan was excellent. However, there is decreased contrast-noise ratio when using 3T scanners [8], which in the hyperacute phase could explain subtle infarcts, such as the one we have reported here, being undetectable. This might mean different DWI parameters could be more appropriate for brainstem infarcts. Despite these clear variations between patients, there is no difference in long term outcomes in DWI negative cases [11].

Acute ischaemic stroke of the brainstem is largely thought to be a result small vessel disease and local branch occlusion $[12,13]$. In this case there was a background of heavy alcohol consumption for the previous 2 years. The risk of stroke is known to rise sharply with heavy alcohol consumption and is considered an age-varying risk factor [14-16]. Furthermore, the rates of atrial fibrillation (AF) increase with even moderate alcohol consumption [17]. This raises the possibility of cardiac thromboembolism when considering the likely aetiology. However, no evidence of AF was captured on prolonged ECG monitoring.

\section{Conclusion}

This case highlights the importance of the history and 
neurological examination as fundamental diagnostic skills in stroke medicine. While neuroimaging continues to advance at a rapid pace, it should still be used as an adjunct to confirm a clinical diagnosis. In the context of acute neurological symptoms conforming to recognised stroke syndromes, if there is a negative finding on initial DWI, particularly if performed early (in the first 6 hours), consideration should be given to repeat imaging after a suitable delay.

\section{References}

1. Gass A, Ay H, Szabo K, Koroshetz WJ. 2004. Diffusion-weighted MRI for the "small stuff": the details of acute cerebral ischaemia. Lancet Neurol 3(1): 39-45. https://doi.org/10.1016/S1474-4422(03)00621-5

2. Broadley SA, Taylor J, Waddy HM, Thompson PD. 2001. The clinical and MRI correlate of ischaemia in the ventromedial midbrain: Claude's syndrome. J Neurol 248(12): 1087-1089. https://doi.org/10.1007/ s004150170030

3. Rathakrishnan R, Sharma VK, Chan BP. 2008. Diffusion-negative MRI in acute ischemic stroke: a case report. Cases J1(1): 65. https://doi. org/10.1186/1757-1626-1-65

4. Jing Z, Zhang S, Tang J, Xu A, Ruan Y, et al. 2014. Hyperacute ischemic stroke without lesions on diffusion-weighted imaging in a patient treated with rtPA thrombolysis. Clin Case Rep 2(3): 70-73. https://doi. org/10.1002/ccr3.60

5. Oppenheim C, Stanescu R, Dormont D, Crozier S, Marro B, et al. 2000. False-negative diffusion-weighted MR findings in acute ischemic stroke. AJNR Am J Neuroradiol 21(8): 1434-1440.

6. Bulut HT, Yildirim A, Ekmekci B, Eskut N, Gunbey HP. 2014. Falsenegative diffusion-weighted imaging in acute stroke and its frequency in anterior and posterior circulation ischemia. J Comput Assist Tomogr 38(5): 627-633. https://doi.org/10.1097/RCT.0000000000000095

7. Lovblad KO, Laubach HJ, Baird AE, Curtin F, Schlaug G, et al. 1998. Clinical experience with diffusion-weighted MR in patients with acute stroke. AJNR Am J Neuroradiol 19(6): 1061-1066.

8. Rosso C, Drier A, Lacroix D, Mutlu G, Pires C, et al. 2010 Diffusion-weighted MRI in acute stroke within the first 6 hours: 1.5 or 3.0 Tesla? Neurology 74(24): 1946-1953. https://doi.org/10.1212/ WNL.0b013e3181e396d1
9. Brisson CD, Hsieh YT, Kim D, Jin AY, Andrew RD. 2014. Brainstem neurons survive the identical ischemic stress that kills higher neurons: insight to the persistent vegetative state. PLoS One 9(5): e96585.https:// doi.org/10.1371/journal.pone.0096585

10. Nouh A, Remke J, Ruland S. 2014. Ischemic posterior circulation stroke: a review of anatomy, clinical presentations, diagnosis, and current management. Front Neurol 5: 30. https://doi.org/10.3389/ fneur.2014.00030

11. Makin SD, Doubal FN, Dennis MS, Wardlaw JM. 2015. Clinically confirmed stroke with negative diffusion-weighted imaging magnetic resonance imaging: longitudinal study of clinical outcomes, stroke recurrence, and systematic review. Stroke 46(11): 3142-3148. https:// doi.org/10.1161/STROKEAHA.115.010665

12. Kim JS, Nah HW, Park SM, Kim SK, Cho KH, et al. 2012. Risk factors and stroke mechanisms in atherosclerotic stroke: intracranial compared with extracranial and anterior compared with posterior circulation disease. Stroke 43(12): 3313-3318. https://doi.org/10.1161/ STROKEAHA.112.658500

13. Lin Y, Zhang L, Bao J, Zhang B, Li H, et al. 2014. Risk factors and etiological subtype analysis of brainstem infarctions. J Neurol Sci 338(12): 118-121. https://doi.org/10.1016/j.jns.2013.12.028

14. Hajat C, Tilling K, Stewart JA, Lemic-Stojcevic N, Wolfe CD. 2004. Ethnic differences in risk factors for ischemic stroke: a European casecontrol study. Stroke 35(7): 1562-1567. https://doi.org/10.1161/01. STR.0000131903.04708.b8

15. Kadlecová P, Andel R, Mikulík R, Handing EP, Pedersen NL. 2015. Alcohol consumption at midlife and risk of stroke during 43 years of follow-up: cohort and twin analyses. Stroke 46(3): 627-633. https://doi. org/10.1161/STROKEAHA.114.006724

16. Zhang C, Qin YY, Chen Q, Jiang H, Chen XZ, et al. 2014. Alcohol intake and risk of stroke: a dose-response meta-analysis of prospective studies. Int J Cardiol 174(3): 669-677. https://doi.org/10.1016/j. ijcard.2014.04.225

17. Larsson SC, Drca N, Wolk A. 2014. Alcohol consumption and risk of atrial fibrillation: a prospective study and dose-response metaanalysis. J Am Coll Cardiol 64(3): 281-289. https://doi.org/10.1016/j. jacc.2014.03.048 